\title{
DIRECT PRODUCTS OF MODULES AND THE PURE SEMISIMPLICITY CONJECTURE. PART II
}

\author{
BIRGE HUISGEN-ZIMMERMANN \\ Department of Mathematics, University of California, Santa Barbara, CA 93106, USA \\ e-mail:birge@math.ucsb.edu \\ and MANUEL SAORÍN \\ Departamento de Matemáticas, Universidad de Murcia, 30100 Espinardo-MU, Spain \\ e-mail:msaorinc@um.es
}

(Received 13 September, 2000; accepted 23 March 2001)

\begin{abstract}
We prove that the module categories of Noether algebras (i.e., algebras module finite over a noetherian center) and affine noetherian PI algebras over a field enjoy the following product property: whenever a direct product $\prod_{n \in \mathbb{N}} M_{n}$ of finitely generated indecomposable modules $M_{n}$ is a direct sum of finitely generated objects, there are repeats among the isomorphism types of the $M_{n}$. The rings with this property satisfy the pure semisimplicity conjecture which stipulates that vanishing one-sided pure global dimension entails finite representation type.
\end{abstract}

2000 Mathematics Subject Classification. 16D70.

This is a follow-up to a joint article of the first-named author with F. Okoh [9], improving significantly on the main result of that note. In rough terms, our aim is to identify the noetherian rings whose module categories display the same productdecomposition properties as have long been observed for finite dimensional algebras. So far, such rings are known to include Artin algebras (this is essentially due to Auslander [2, Corollary 3.2]) and commutative noetherian domains of Krull dimension 1 (as was proved in [9]). As we show here, not only can all requirements on the Krull dimension be dropped, but rudimentary commutativity conditions already guarantee the desired product-behavior.

The principal motivation for our interest in decomposition properties of direct products lies in the fact that they massively impinge on global decomposition patterns within the pertinent module categories (see for example [6], [16], [10]). So, in particular, it is the lack of understanding of direct products which is responsible for the fact that it is still unresolved whether the rings whose left modules split into finitely generated submodules (the left pure semisimple rings) are necessarily of finite representation type. For a synopsis of the extensive history of this pursuit, going back to the work of Koethe in the 1930's and of Cohen-Kaplansky in the early 1950's ([12], [4]), the reader is referred to [11], [15], or [8]. The following question crystallizes the remaining difficulties on the road towards a full resolution of the 'pure semisimplicity conjecture' (which, at this point, is believed to fail in general).

Central problem. For which rings $R$ (associative and with identity) does the following hold? If $\left(M_{n}\right)_{n \in \mathbb{N}}$ is any sequence of pairwise non-isomorphic finitely generated, 
indecomposable left $R$-modules, the direct product $\prod_{n \in \mathbb{N}} M_{n}$ is not a direct sum of finitely generated submodules.

In this form, the question was raised by Okoh. It connects with the pure semisimplicity problem as follows. Whenever a class of rings is known to satisfy the specified product condition, all left pure semisimple members of that class have finite representation type. In our opinion, this problem also holds independent interest as a yardstick measuring the current level of understanding of submodule lattices of direct products.

In [9] it was shown that commutative noetherian domains of Krull dimension 1 satisfy the above product condition. This led to the conjecture that the same holds true for arbitrary noetherian PI-rings, which is in keeping with the fact that the left pure semisimple artinian PI-rings have finite representation type (see [7] and [14]). Here we come close to confirming it. Namely, we establish the product condition for arbitrary Noether algebras (rings which are module finite over a noetherian center), as well as for affine noetherian PI-rings (that is, affine noetherian PI algebras over $\mathbb{Z}$ ).

For the slightly more general statement of our theorem, we recall that a commutative ring is a Jacobson ring if each prime ideal is an intersection of maximal ideals. So, in particular, affine noetherian PI-algebras over fields or over noetherian domains of Krull dimension 1 fall into the second class of rings addressed by the following theorem.

TheOREM. Suppose that $R$ is either a Noether algebra, or else an affine noetherian $P I$-algebra over a noetherian Jacobson ring. Then $R$ satisfies the product condition of our 'central problem'; that is, given any sequence $\left(M_{n}\right)_{n \in \mathbb{N}}$ of pairwise non-isomorphic finitely generated, indecomposable left $R$-modules, the direct product $\prod_{n \in \mathbb{N}} M_{n}$ fails to be a direct sum of finitely generated components.

Proof. Our strategy consists of playing the problem back to Artin algebras, where it is already resolved. To that end, we verify in an initial step that both classes of rings in our claim enjoy the following property: for any sequence $P_{1}, \ldots, P_{n}$ of (not necessarily distinct) left primitive ideals of $R$, the factor ring $R /\left(P_{1} \ldots P_{n}\right)$ is an Artin algebra.

First suppose that $R$ is a Noether algebra and so, in particular, a PI-ring. Denote the center of $R$ by $C$. If $P \subseteq R$ is a left primitive ideal, Kaplansky's Theorem guarantees $P \cap C$ to be a maximal ideal of $C$. Indeed, $R / P$ is a finite dimensional algebra over a central subfield $K$ containing $C /(P \cap C)$, and since the embedding $C /(P \cap C) \hookrightarrow K$ is integral, $C /(P \cap C)$ is a field as well. Hence, given any finite sequence $P_{1}, \ldots, P_{n}$ of left primitive ideals of $R$, the factor ring $C /\left(\left(P_{1} \cap C\right) \cdots\left(P_{n} \cap C\right)\right)$ has Krull dimension zero and is therefore artinian. So is, a fortiori, the central subring $C /\left(\left(P_{1} \ldots P_{n}\right) \cap C\right)$ of $R /\left(P_{1} \ldots P_{n}\right)$.

Next suppose that $C$ is a central noetherian Jacobson subring of $R$. We will use the following upgrade of Kaplansky's Theorem, which is due to Amitsur and Procesi ([1], see also [13] or [5] for a slick proof due to Duflo): if $R$ is an affine PI-algebra over $C$ and $P$ any left primitive ideal of $R$, then $P \cap C$ is a maximal ideal of $C$, and $R / P$ is a simple finite dimensional $(C /(P \cap C))$-algebra. Now suppose that $R$ is noetherian in addition, and let $P_{1}, \ldots, P_{n}$ be left primitive ideals of $R$. Since the successive factors of the chain $R \supseteq P_{n} \supseteq P_{n-1} P_{n} \supseteq \ldots \supseteq\left(P_{1} \cdots P_{n}\right)$ are finitely generated left modules over the rings $R / P_{i}$ and these rings, in turn, are module-finite 
over $C$, we see that $R /\left(P_{1} \ldots P_{n}\right)$ is module-finite over $C /\left(\left(P_{1} \ldots P_{n}\right) \cap C\right)$, the latter ring being a homomorphic image of $C /\left(\left(P_{1} \cap C\right) \ldots\left(P_{n} \cap C\right)\right)$ and hence artinian.

The theorem will follow from the following lemma.

Lemma. Suppose that $R$ is a two-sided noetherian ring with the property that, for any finite sequence $P_{1}, \ldots, P_{n}$ of (not necessarily distinct) left primitive ideals, the factor ring $R /\left(P_{1} \ldots P_{n}\right)$ is an Artin algebra. Then $R$ satisfies the conclusion of the theorem above.

Proof. Let $\left(M_{n}\right)_{n \in \mathbb{N}}$ be a sequence of finitely generated indecomposable left $R$-modules with $M_{i} \neq M_{j}$ whenever $i \neq j$. Observe that, for any nonzero finitely generated left $R$-module $X$, there exists a left primitive ideal $P \subseteq R$ with $P X \varsubsetneqq X$. Indeed, if $Y$ is a maximal submodule of $X$, the conductor ideal $P=[Y: X]$ satisfies these requirements.

We now apply this observation to finitely generated left $R$-modules of the form $A M_{n}$, where $A$ is a two-sided ideal. Our goal is to construct a descending chain $\left(A_{n}\right)_{n \in \mathbb{N}}$ of ideals, each term of which is a finite product of left primitive ideals, with the following property: for $n \in \mathbb{N}$, either $A_{i} M_{n}=0$, or else there exists an integer $j>i$ such that $A_{j} M_{n} \subsetneq A_{i} M_{n}$.

For that purpose, we will follow a diagonal procedure involving 'zigzags of increasing amplitude'. If $M_{1}=0$, let $P_{1}$ be any left primitive ideal; otherwise, pick $P_{1}$ left primitive with $P_{1} M_{1} \varsubsetneqq M_{1}$, and set $A_{1}=P_{1}$. If $A_{1} M_{2}=0$, set $A_{2}=A_{1}$; otherwise, choose a left primitive ideal $P_{2}$ with $P_{2} A_{1} M_{2} \varsubsetneqq A_{1} M_{2}$, and set $A_{2}=P_{2} A_{1}$. If $A_{2} M_{1}=0$, set $A_{3}=A_{2}$; otherwise, pick a left primitive ideal $P_{3}$ with $P_{3} A_{2} M_{1} \varsubsetneqq A_{2} M_{1}$, and set $A_{3}=P_{3} A_{2}$. In the next step, we define $A_{4}=P_{4} A_{3}$, where $P_{4}$ is a left primitive ideal such that $P_{4} A_{3} M_{2} \varsubsetneqq A_{3} M_{2}$, unless $A_{3} M_{2}=0$, and in the latter case we set $A_{4}=A_{3}$. Now we move on to $M_{3}$, cutting down the size of the left module $A_{4} M_{3}$ if it is nonzero, then we return to $M_{1}, M_{2}, M_{3}$, from whence we move up to $M_{4}$, following the pattern

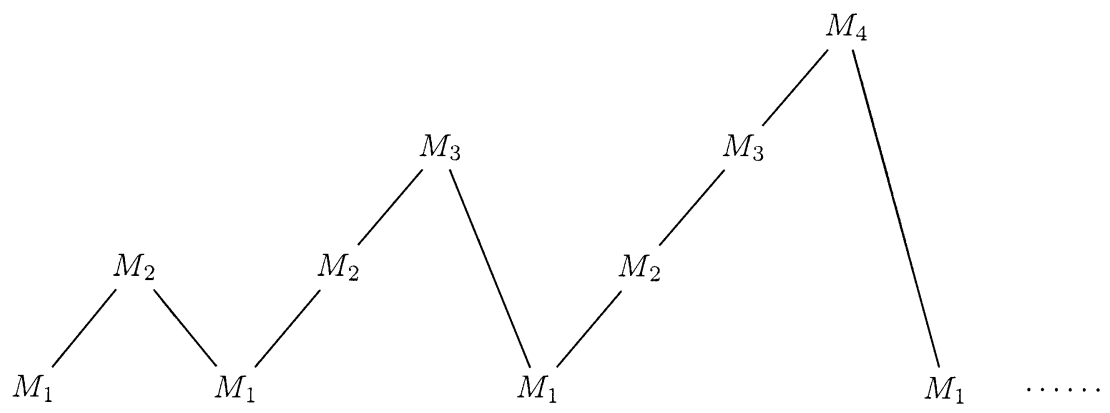

Inductively, this process yields a chain of ideals $A_{1} \supseteq A_{2} \supseteq \ldots$ conforming to the above requirements.

Since all of the $A_{n}$ are finitely generated right ideals of $R$, the following assignments yield $p$-functors in the sense of [16]; that is subfunctors of the forgetful functor $R$-Mod $\rightarrow \mathbb{Z}$-Mod which commute with direct products: 


$$
F_{n}: R \text {-Mod } \rightarrow \mathbb{Z} \text {-Mod, } \quad X \mapsto A_{n} X .
$$

In the present situation, we are actually dealing with subfunctors $F_{n}$ of the identity functor $R$-Mod $\rightarrow R$-Mod taking finitely generated left $R$-modules to finitely generated left $R$-modules. Set $M=\prod_{n \in \mathbb{N}} M_{n}$, and assume that, to the contrary of our claim, this product splits into finitely generated direct summands, say

$$
M=\bigoplus_{l \in L} B_{l}
$$

where each $B_{l}$ is a finitely generated left $R$-module. An upgraded version of Chase's Lemma [8, Lemma 11] (cf [3, Theorem 1.2] for the original result) then yields a natural number $n_{0}$ and a finite subset $L_{0} \subseteq L$ such that

$$
\prod_{n \geq n_{0}} F_{n_{0}} M_{n} \subseteq \bigoplus_{l \in L_{0}} F_{n_{0}} B_{l}+\bigcap_{k \in \mathbb{N}} F_{k} M .
$$

Case 1: $F_{n_{0}} M_{n}=0$ for all but finitely many $n \in \mathbb{N}$. Set $N_{0}=\left\{n \in \mathbb{N} \mid F_{n_{0}} M_{n}=0\right\}$ and $N_{1}=\mathbb{N} \backslash N_{0}$. Factoring $F_{n_{0}} M$ out of both sides of equality (1) yields

$$
\prod_{n \in N_{0}} M_{n} \oplus \prod_{n \in N_{1}}\left(M_{n} / F_{n_{0}} M_{n}\right)=\bigoplus_{l \in L}\left(B_{l} / F_{n_{0}} B_{l}\right) .
$$

Observe that the summand $D:=\prod_{n \in N_{1}}\left(M_{n} / F_{n_{0}} M_{n}\right)$ on the left-hand side of this last equality is a finitely generated $R$-module, since $N_{1}$ is finite. It is therefore contained in some finite subsum $B:=\bigoplus_{l \in L_{1}}\left(B_{l} / F_{n_{0}} B_{l}\right)$ of the right-hand direct sum, which is in turn finitely generated over $R$. In summary, we thus obtain

$$
\prod_{n \in N_{0}} M_{n} \cong \bigoplus_{l \in L \backslash L_{1}}\left(B_{l} / F_{n_{0}} B_{l}\right) \oplus B / D
$$

where all summands on the right are finitely generated $R$-modules. Observe that all of the modules involved in this isomorphism are annihilated by $A_{n_{0}}$, and so are modules over $R / A_{n_{0}}$, the latter being an Artin algebra by hypothesis. But this is incompatible with the fact that our claim is known to hold for such algebras. Indeed, the infinite family $\left(M_{n}\right)_{n \in N_{0}}$ consists of pairwise non-isomorphic indecomposable $R / A_{n_{0}}$-modules, since their $R / A_{n_{0}}$-structure coincides with their $R$-structure. This rules out the first case.

Case 2: $F_{n_{0}} M_{n} \neq 0$ for infinitely many $n \in \mathbb{N}$. We denote by $N_{1}$ the infinite set $\left\{n \in \mathbb{N} \mid n \geq n_{0}\right.$ and $\left.F_{n_{0}} M_{n} \neq 0\right\}$. Next we factor the term $\bigcap_{k \in \mathbb{N}} F_{k} M$ equal to $\prod_{n \in \mathbb{N}}\left(\bigcap_{k \in \mathbb{N}} F_{k} M_{n}\right)$ out of both sides of the inclusion labeled (2) to obtain

$$
\prod_{n \geq n_{0}}\left(F_{n_{0}} M_{n} / \bigcap_{k \in \mathbb{N}} F_{k} M_{n}\right) \subseteq \bigoplus_{l \in L_{0}}\left(F_{n_{0}} B_{l} / \bigcap_{k \in \mathbb{N}} F_{k} B_{l}\right) .
$$

In justifying this inclusion, keep in mind that $p$-functors automatically commute with direct sums. We note that the direct product on the left-hand side equals

$$
\prod_{n \in N_{1}}\left(F_{n_{0}} M_{n} / \bigcap_{k \in \mathbb{N}} F_{k} M_{n}\right) \text {. }
$$


Now $\bigcap_{k \in \mathbb{N}} F_{k} M_{n} \varsubsetneqq F_{n_{0}} M_{n}$ for all $n \in N_{1}$ by construction of the functors $F_{k}$, and consequently, the left-hand side of the inclusion we just derived is not finitely generated; this is due to the fact that it contains the infinite direct sum $\bigoplus_{n \in N_{1}}\left(F_{n_{0}} M_{n} / \bigcap_{k \in \mathbb{N}} F_{k} M_{n}\right)$. But the right-hand side of our inclusion is finitely generated, and we have again reached a contradiction.

This completes the proof of the lemma.

\section{REFERENCES}

1. S. A. Amitsur and C. Procesi, Jacobson rings and Hilbert algebras with polynomial identities, Ann. Mat. Pura Appl. 71 (1966), 61-72.

2. M. Auslander, Large modules over Artin algebras, in Algebra, topology and categories (Academic Press, 1976), 1-17.

3. S. U. Chase, Direct products of modules, Trans. Amer. Math. Soc. 97 (1960), 457473.

4. I. S. Cohen and I. Kaplansky, Rings for which every module is a direct sum of cyclic modules, Math. Z. 54 (1951), 97-101.

5. M. Duflo, Certaines algèbres de type fini sont des algèbres de Jacobson, J. Algebra 27 (1973), 358-365.

6. L. Gruson and C. U. Jensen, Deux applications de la notion de L-dimension, C.R. Acad. Sci. Paris, Sér. A 282 (1976), 23-24.

7. I. Herzog, A test for finite representation type, J. Pure Appl. Algebra 95 (1994), 151-182.

8. B. Huisgen-Zimmermann, Purity, algebraic compactness, direct sum decompositions, and representation type, in Infinite Length Modules (Bielefeld 1998) (H. Krause, H. Lenzing and C. M. Ringel, eds.) (Birkhaüser, 2000), 331-367.

9. B. Huisgen-Zimmermann and F. Okoh, Direct products of modules and the pure semisimplicity conjecture, Comm. Algebra 29 (2001), 271-276.

10. B. Huisgen-Zimmermann and W. Zimmermann, Algebraically compact rings and modules, Math. Z. 161 (1978), 81-93.

11. B. Huisgen-Zimmermann and W. Zimmermann, On the sparsity of representations of rings of pure global dimension zero, Trans. Amer. Math. Soc. 320 (1990), 695-711.

12. G. Koethe, Verallgemeinerte abelsche Gruppen mit hyperkomplexem Operatorenring, Math. Z. 39 (1935), 31-44.

13. L. H. Rowen, Ring theory II (Academic Press, 1988).

14. M. Schmidmeier, The local duality for homomorphisms and an application to pure semisimple PI-rings, Colloq. Math. 77 (1998), 121-132.

15. D. Simson, Dualities and pure semisimple rings, in Abelian groups, module theory, and topology (Padova 1997) (D. Dikranjan and L. Salce, eds.) Lecture Notes in Pure and Appl. Math., Vol. 201 (Dekker, New York, 1988), 381-388.

16. W. Zimmermann, Rein-injektive direkte Summen von Moduln, Comm. Algebra 5 (1977), 1083-1117. 